BMJ Open Diabetes Research \& Care

\title{
Clinical and economic outcomes among injection-naïve patients with type 2 diabetes initiating dulaglutide compared with basal insulin in a US real-world setting: the DISPEL Study
}

\author{
Reema Mody, ${ }^{1}$ Qing Huang, ${ }^{2}$ Maria Yu, ${ }^{3}$ Hiren Patel, ${ }^{4}$ Xian Zhang, ${ }^{2}$ Liya Wang, ${ }^{2}$ \\ Michael Grabner (1) ${ }^{2}$
}

\begin{abstract}
To cite: Mody R, Huang $Q$, Yu M, et al. Clinical and economic outcomes among injection-naïve patients with type 2 diabetes initiating dulaglutide compared with basal insulin in a US real-world setting: the DISPEL Study. BMJ Open Diab Res Care 2019;7:e000884. doi:10.1136/ bmjdrc-2019-000884
\end{abstract}

- Additional material is published online only. To view please visit the journal online (http://dx.doi.org/10.1136/ bmjdrc-2019-000884).

Selected content was presented at the 2019 American Diabetes Association Annual Meeting and the 2019 ISPOR Annual International Meeting.

Received 13 September 2019 Accepted 1 October 2019

Check for updates

(c) Author(s) (or their employer(s)) 2019. Re-use permitted under CC BY-NC. No commercial re-use. See rights and permissions. Published by BMJ.

For numbered affiliations see end of article.

Correspondence to Dr Reema Mody; mody_reema@lilly.com

\section{ABSTRACT}

Aims To report 1-year clinical and economic outcomes from the retrospective DISPEL (Dulaglutide vs Basal InSulin in Injection Naïve Patients with Type 2 Diabetes: Effectiveness in ReaL World) Study.

Materials and methods This observational claims study included patients with type 2 diabetes (T2D) and $\geq 1$ claim for dulaglutide or basal insulin between November 2014 and April 2017 (index date=earliestfill date). Propensity score matching was used to address treatment selection bias. Change from baseline in hemoglobin $\mathrm{A} 1 \mathrm{c}(\mathrm{HbA1C})$ was compared between the matched cohorts using analysis of covariance; diabetes-related costs were analyzed using generalized linear models.

Results Matched cohorts ( 903 pairs total; 523 pairs with complete cost data) were balanced in baseline characteristics with mean $\mathrm{HbA1c} 8.6 \%$, mean age 54 years. At 1 year postindex, dulaglutide patients had significantly greater reduction in $\mathrm{HbA} 1 \mathrm{c}$ than basal insulin $(-1.12 \%$ vs $-0.51 \%, p<0.01)$, lower medical costs $(\$ 3753$ vs $\$ 7604, p<0.01$ ), higher pharmacy costs ( $\$ 9809$ vs $\$ 6175, p<0.01)$, and similar total costs ( $\$ 13562$ vs $\$ 13$ $779, \mathrm{p}=0.76$ ). Medical and total costs per $1 \% \mathrm{HbA} 1 \mathrm{c}$ reduction were lower for dulaglutide than basal insulin (medical: \$3128 vs \$12 673, p<0.01; total: \$11302 vs $\$ 22965, p<0.01$ ), while pharmacy costs per $1 \%$ $\mathrm{HbA} 1 \mathrm{c}$ reduction were lower without reaching statistical significance ( $\$ 8174$ vs $\$ 10292, p=0.15$ ).

Conclusions In this real-world study, patients with T2D initiating dulaglutide demonstrated greater $\mathrm{HbA1C}$ reduction compared with those initiating basal insulin. Although total diabetes-related costs were similar, the total diabetes-related costs per $\mathrm{HbA} 1 \mathrm{c}$ reduction were lower for dulaglutide, highlighting the importance of evaluating effectiveness along with the economic impact of medications.

\section{INTRODUCTION}

The majority of patients with type 2 diabetes (T2D) have to initiate injectable therapies for better glycemic control after failure of oral antidiabetic drugs (OADs) along with lifestyle changes. ${ }^{12}$ Current American Diabetes
Significance of this study

What is already known about this subject?

- Dulaglutide, a once-weekly glucagon-like peptide 1 receptor agonist, has demonstrated superior glycemic control over daily insulin glargine in controlled clinical trial settings.

Despite this, basal insulins continue to be used by $>70 \%$ of patients with type 2 diabetes requiring additional glycemic control after oral therapies.

- Studies comparing the real-world effectiveness among patients initiating dulaglutide versus basal insulin as their first injectable therapy are lacking.

What are the new findings?

- To our knowledge, this is the first study comparing real-world effectiveness of patients initiating dulaglutide or basal insulin as their first injectable antidiabetic treatment.

- After 1 year, patients initiating on dulaglutide had significantly greater reductions in $\mathrm{HbA1C}$ compared with matched patients initiating on basal insulin.

- The dulaglutide group had lower medical costs but higher pharmacy costs than the basal insulin group, while total costs were similar between the two groups. The total cost per $1 \% \mathrm{HbA} 1 \mathrm{c}$ reduction was lower for dulaglutide versus basal insulin.

How might these results change the focus of research or clinical practice?

- In this real-world population, dulaglutide demonstrated significantly greater and clinically meaningful $\mathrm{HbA1C}$ reduction compared with similar patients initiating basal insulin at 1-year follow-up.

- These findings along with prior comparative data generated in a clinical trial setting will help clinical practitioners make evidence-based choices of either dulaglutide or basal insulin as the first injectable agent after oral medications.

- The results on the cost per $1 \% \mathrm{HbA} 1 \mathrm{c}$ reduction also underscore the importance of evaluating effectiveness and economic impact of medications together. 
Association (ADA) Standards of Care recommend glucagon-like peptide 1 receptor agonists (GLP-1 RAs) before basal insulin for most patients who need injectable therapy. ${ }^{1}$ Unlike basal insulin, GLP-1 RAs have a multifaceted mechanism of action, lowering both fasting and postprandial glucose levels while curbing appetite and reducing caloric intake leading to clinically relevant weight loss. ${ }^{3}$ The overall glycemic control obtained by GLP-1 RAs-particularly with long-acting agentsis slightly better than that achieved with basal insulin. ${ }^{3}$ Moreover, 'psychological insulin resistance' seen among patients and physicians is the leading cause of clinical inertia when introducing injectable therapies in patients with T2D. ${ }^{4}$ Due to their lower injection burden, clinical benefits and low risk of hypoglycemia, ${ }^{35}$ GLP-1 RAs, especially the weekly agents, may help overcome this clinical inertia with first injection while potentially improving adherence and compliance over daily insulin injections.

Despite these advantages of GLP-1 RAs, and ADA recommending them to be used as first-line injectable agents, basal insulin continues to be used in approximately $70 \%$ of patients with T2D who require injectable medication. ${ }^{36}$ Randomized clinical trials have shown greater hemoglobin Alc (HbAlc) and weight reduction with GLP-1 RAs compared with basal insulin in injectionnaïve patients; ${ }^{3}$ a limited number of real-world comparative effectiveness studies have shown generally similar findings. ${ }^{78}$

Dulaglutide is a once-weekly GLP-1 RA indicated as an adjunct to diet and exercise to improve glycemic control in adults with T2D. Previous randomized clinical trials have demonstrated the safety and effectiveness of dulaglutide. ${ }^{9-11}$ In patients with T2D who were injectionnaïe, dulaglutide was associated with greater reductions in HbAlc levels and weight loss as compared with insulin glargine, in addition to lower rates of hypoglycemia. ${ }^{12} 13$ To our knowledge, there is no published study comparing real-world clinical outcomes of patients with T2D initiating basal insulin versus dulaglutide. In addition to clinical outcomes, costs are a consideration for the selection of these treatments, and therefore a better understanding of economic outcomes and cost-effectiveness is warranted. The current DISPEL (Dulaglutide vs Basal InSulin in Injection Naïve Patients with Type 2 Diabetes: Effectiveness in ReaL World) Study examined glycemic control, healthcare costs, and costs per $1 \% \mathrm{HbA1c}$ reduction in patients newly initiating dulaglutide versus basal insulin over a 1-year period.

\section{METHODS}

This was a retrospective observational cohort study using longitudinal pharmacy and medical claims data augmented with electronic laboratory results. Claims data for the study period (May 1, 2014 through April 30, 2018) were obtained from the HealthCore Integrated Research Database (HIRD). The HIRD contains health plan claims integrated across data sources and places of service (ie, professional claims, facility claims, outpatient pharmacy claims, outpatient laboratory results, and enrolment information) as well as across years (from 2006 through the most recent calendar quarter). Data are geographically diverse and obtained from a range of commercial health plans across all regions of the USA.

\section{Patient identification}

Patients were identified from the HIRD based on their medical and pharmacy claims data. Patients with at least one outpatient pharmacy claim for dulaglutide or basal insulin (insulin glargine, insulin detemir, or insulin degludec in any concentration) during the index period (November 1, 2014 through April 30, 2017) were selected. The index date was defined as the earliest occurrence of a prescription fill for dulaglutide or basal insulin during the index period. Patients were assigned to either the dulaglutide or basal insulin treatment cohorts depending on the index medication; all analyses were conducted on an intent-to-treat basis.

All patients participating in the study were adults (18 years or older on the index date) with $\geq 6$ months preindex (baseline) and $\geq 12$ months postindex (follow-up) continuous medical and pharmacy enrolment. Patients had at least one claim for T2D during the baseline period. All patients were required to be enrolled in commercial or Medicare Advantage health insurance plans during the baseline and postindex periods, and to have at least one $\mathrm{HbA1c}$ lab result at baseline (range 183 days before index date to 14 days after) and at least one HbAlc lab result at the 1-year postindex period (range 275 days to 410 days after the index date); if multiple lab results were available in each time period, the one closest to index date/index date +365 days was selected for analysis.

Patients were excluded from the study if they had any antidiabetic injectable during the 6 months baseline period (in order to identify injection-naïve patients) or if they had claims for any antidiabetic injectable other than dulaglutide (for those in the dulaglutide cohort) or basal insulin (for those in the basal insulin cohort) on the index date. Additionally, patients with at least one medical claim with a diagnosis of type 1 diabetes or secondary diabetes during the baseline period, a diagnosis of pregnancy or childbirth-related diabetes during the entire study period, or bariatric surgery during the entire study period were also excluded. Patients with incomplete pharmacy cost capture were excluded from the cost-related portion of the study.

\section{Outcome measures}

Patient demographic and clinical characteristics, including prescribing physician specialty, were assessed on the index date. Baseline comorbidities of interest were identified based on International Classification of Diseases, Ninth/ Tenth Revisions, Clinical Modification (ICD-9/10CM) diagnosis codes (see online appendix table 1). The QuanCharlson Comorbidity Index ${ }^{14}$ and the adapted Diabetes Complications Severity Index (aDCSI) ${ }^{15}$ were calculated 
and reported. Diabetes medication use was also captured, including OADs (ie, metformin, thiazolidinediones, sulfonylureas, dipeptidyl peptidase-4 inhibitors, and sodiumglucose co-transporter-2 (SGLT2) inhibitors), basal and non-basal insulins, and GLP-1 RAs (insulins and GLP-1 RAs during the postindex period only).

Measures of glycemic control included change in HbA1c from baseline to 1-year follow-up and percentage of patients reaching $\mathrm{HbAlc}$ target of $<7 \%$ or $<8 \%$ at 1-year follow-up. These outcomes were also assessed in two clinically relevant subgroups based on baseline age (patients 65 years of age or older) and HbAlc levels (greater than 9\%). Additional glycemic control measures included HbAlc levels at 3-month, 6-month, 9-month, and 12-month postindex time points to investigate longitudinal changes.

Costs were calculated as the sum of patient-paid costs, plan-paid costs, and coordination of benefits, adjusted to 2017 USD price levels. All-cause medical/pharmacy costs represent aggregated costs for any medical/pharmacy claims; total costs are the sum of medical and pharmacy costs. Diabetes-related medical costs represent aggregated costs for any medical claims that had diagnosis codes for diabetes; diabetes-related pharmacy costs are summed costs for any pharmacy claims for antidiabetic medications (OADs and injectables).

All-cause and diabetes-related costs incurred per $1 \%$ HbA1c reduction were calculated using the following formula: mean 1year follow-up costs divided by mean percentage reduction in $\mathrm{HbAlc}$ from baseline to 1 year follow-up (ie, baseline HbAlc percentage minus 1year follow-up HbA1c percentage).

\section{Statistical analysis}

All variables were summarized using descriptive statistics. Mean, SD, and median were reported for continuous variables and counts and percentages for categorical variables. T-tests and chi-square tests were used to compare continuous and categorical variables, respectively. For costs, both t-tests and a generalized linear model with $\gamma$ distribution and $\log$ link function with cohort as the independent variable were used.

Due to the observational nature of the study and the lack of randomization of patients into the two study cohorts, it was necessary to account for treatment selection bias. We used exact matching on baseline HbAlc category $(<7 \%, 7$ to $<8 \%$, etc.) combined with propensity score (PS) matching, with the PS defined as the probability of initiating dulaglutide (vs basal insulin) given the baseline patient characteristics and estimated using logistic regression (see footnote for table 1 for a list of variables included in the PS regression). Matching was finalized before the outcome analyses were conducted. Nearest neighbor greedy matching with caliper was used, with the caliper width set to 0.2 of the SD of the logit of the PS. Absolute standardized differences of $\leq 0.1$ were considered to denote balance in baseline characteristics between the cohorts. ${ }^{16}$
Analysis of covariance with baseline HbA1c level (a continuous variable) as the covariate was used to compare the change in $\mathrm{HbAlc}$ between the two cohorts. Bootstrap sampling with 5000 replications was used to obtain an estimate of the SE of the mean and $95 \%$ CI for the ratio of cost per $1 \%$ HbAlc reduction. ${ }^{17}$ HbAlc changes over time were analyzed through a mixed-effects model with repeated measurements using restricted maximum likelihood with treatment, time, and treatment-by-time interaction as fixed effects, baseline $\mathrm{HbA1c}$ as covariate, and patient as random effect. ${ }^{18}$ An unstructured covariance matrix was used to model the within-patient errors.

An $\alpha$ level of 0.05 was used to identify statistical significance. No adjustments for multiple comparisons were made in this study. The 6-month baseline costs were annualized to allow comparison with the 12-month follow-up costs.

\section{RESULTS}

\section{Baseline characteristics}

A total of 4296 patients (1103 initiating dulaglutide, 3193 initiating basal insulin) were identified from the HIRD. After application of inclusion/exclusion criteria and matching, each treatment cohort contained 903 patients, of which 523 pairs had complete pharmacy cost data (online appendix figure 1).

Demographics, baseline clinical characteristics, and antidiabetic utilization were evaluated for both the prematching (online appendix table 2) and postmatching populations (table 1). Before matching, notable betweengroup differences were observed for prescribing specialty (29\% endocrinologist for dulaglutide vs $16 \%$ for basal insulin), aDCSI score (mean 0.6 vs 0.9 ), obesity (32\% vs $22 \%$ ), use of certain OADs (metformin: $79 \%$ vs $69 \%$; sulfonylureas: $35 \%$ vs $46 \%$; SGLT2: $28 \%$ vs $13 \%$ ) and number of OAD fills (4.8 vs 4.1). Moreover, before matching baseline HbAlc levels were lower in the dulaglutide cohort (mean 8.37 vs 9.86 ; $\%$ with $\mathrm{HbAlc}<7 \%$ : $20 \%$ vs $6 \%$ ).

After matching, patient and clinical characteristics including HbAlc levels were balanced between the two treatment cohorts (all standardized differences $\leq 0.1$; table 1). The mean age was 54 years and approximately half of the population was male. Index prescriptions most commonly were from primary care physicians (50\%), followed by endocrinologists $(25 \%)$. The most common baseline comorbidities in both treatment groups were dyslipidemia ( $76 \%$ dulaglutide, $75 \%$ basal insulin), hypertension ( $74 \%$ dulaglutide, $75 \%$ basal insulin), and obesity (28\% both groups). The majority of patients $(90 \%)$ used an OAD at baseline. Mean HbAlc levels were 8.65 vs 8.64, and $13 \%$ of patients had $\mathrm{HbAlc}<7 \%$.

\section{Postindex medication use}

During the 1-year postindex period, $11 \%$ of those in the dulaglutide cohort had fills for basal insulin. In the basal insulin cohort, $10 \%$ had fills for any GLP-1 RA out of which $4 \%$ had fills for dulaglutide (online appendix table 3). A 
Table 1 Postmatching patient ${ }^{\star}$ demographic and clinical characteristics

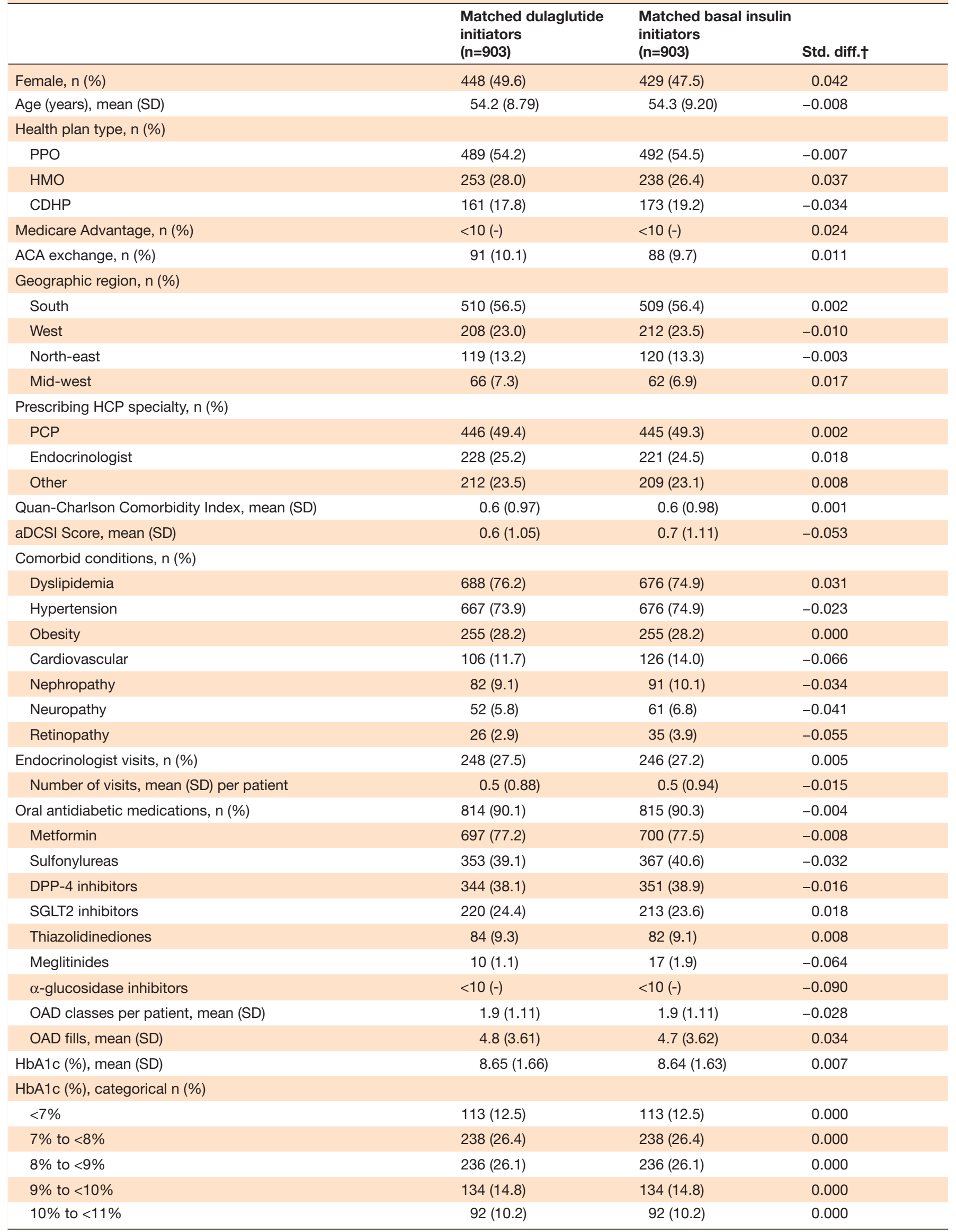




\begin{tabular}{|c|c|c|c|}
\hline & $\begin{array}{l}\text { Matched dulaglutide } \\
\text { initiators } \\
(n=903)\end{array}$ & $\begin{array}{l}\text { Matched basal insulin } \\
\text { initiators } \\
(n=903)\end{array}$ & Std. diff.† \\
\hline$\geq 11 \%$ & $90(10.0)$ & $90(10.0)$ & 0.000 \\
\hline
\end{tabular}

*Patients were matched using propensity scores. Exact matching was also applied on categories of baseline HbA1c and whether a patient had complete costs. Propensity scores were calculated via logistic regression using the covariates age (continuous as well as age $\geq 65$ vs age $<65$ ), gender, geographic location, Affordable Care Act exchange coverage, health plan type and prescribing healthcare provider specialty (endocrinologist vs PCP vs others/missing) on the index date; and aDCSI (continuous and categorical), presence of cardiovascular disease, presence of obesity, presence of peripheral vascular disorders, presence of renal diseases, presence of retinopathy, presence of cerebrovascular disease, presence of neuropathy, presence and number of endocrinologist visits, presence of all-cause inpatient hospitalization, presence of all-cause ER visit, all-cause hospitalization LOS, number of diabetes-related prescription drug fills, presence of OAD fills, number of OAD medication classes (continuous and categorical), presence of metformin, sulfonylureas, SGLT2 inhibitors, diabetes supplies, HbA1c results, and all-cause total medical costs ( $<100 \mathrm{~K}$ vs $100 \mathrm{~K}$ to $<200 \mathrm{~K}$ vs $200 \mathrm{~K}$ to $<500 \mathrm{~K}$ vs $\geq 500 \mathrm{~K}$ ) during the baseline period.

†Standardized differences: absolute standardized differences of $\leq 0.10$ were considered to denote balance in baseline characteristics between the cohorts. All $p$ values $>0.05$.

ACA, Affordable Care Act; aDCSI, adapted Diabetes Complications Severity Index; CDHP, consumer-driven health plan; DPP-4, dipeptidyl peptidase-4; ER, emergency room; HCP, healthcare provider; HMO, health maintenance organization; LOS, length of stay; OAD, oral antidiabetic drug; PCP, primary care physician; PPO, preferred provider organization; SGLT2, sodium-glucose co-transporter-2.

smaller proportion of patients in the dulaglutide cohort versus the basal insulin cohort had fills for non-basal insulin (3\% vs $17 \%)$.

\section{Glycemic control}

In the matched population, patients in the dulaglutide cohort experienced a mean reduction of $1.12 \%$ in $\mathrm{HbAlc}$ levels from baseline to 1 year postindex, compared with a $0.51 \%$ reduction in the basal insulin cohort $(\mathrm{p}<0.001$; figure 1 and online appendix table 4). Additionally,

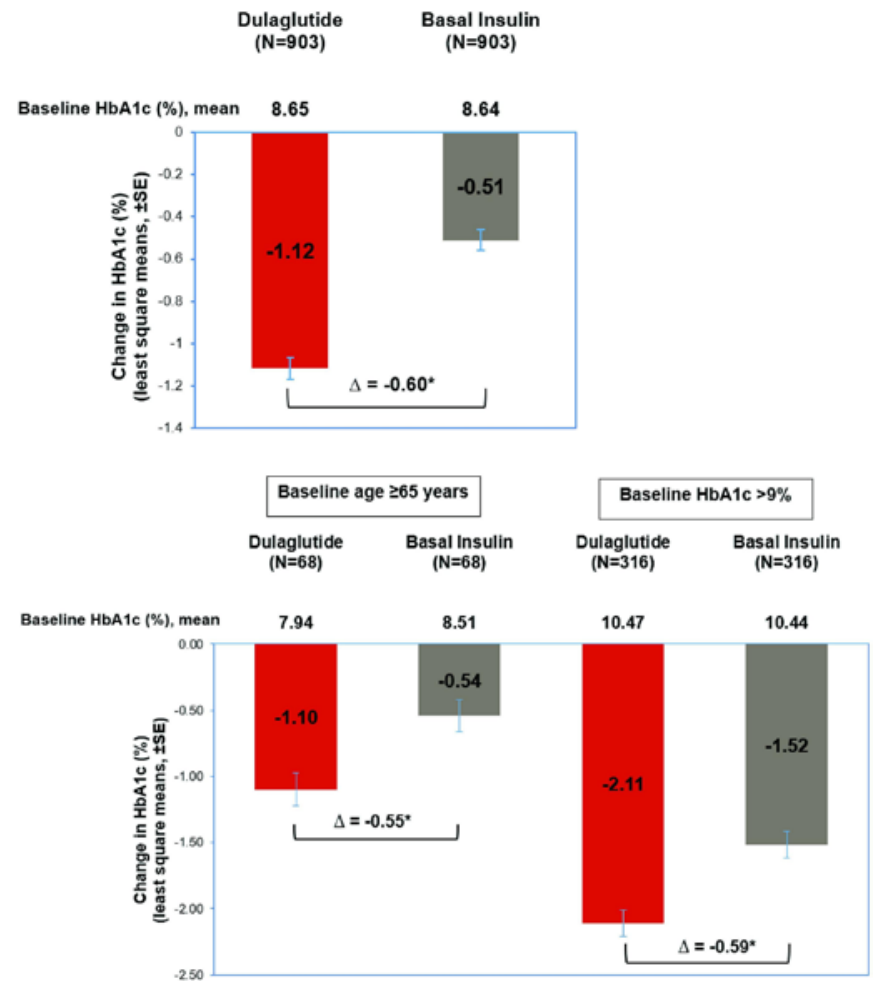

Figure 1 Glycemic outcomes at 1 year postindex among patients initiating dulaglutide versus basal insulin.

*Significant difference between dulaglutide versus basal insulin with $p$ value $<0.05$. Estimates obtained using ANCOVA with baseline HbA1c level (continuous variable) as covariate. significantly more patients in the dulaglutide cohort achieved either a reduction of at least $1 \%$ in HbAlc or an $\mathrm{HbAlc}$ level less than $7 \%$ than in the basal insulin cohort $(66 \%$ vs $45 \%, \mathrm{p}<0.001$; online appendix table 4). The HbAlc distribution in the dulaglutide cohort noticeably shifted from baseline to postindex to reflect a larger proportion of patients at lower HbAlc categories; this effect was not observed in the basal insulin cohort (online appendix figure 2).

In the subgroup of patients with a baseline HbA1c level $>9 \%$ or a baseline age $\geq 65$ years, patients receiving dulaglutide also showed greater improvements in glycemic control, as measured by HbA1c levels, compared with those initiating basal insulin. Among patients whose baseline HbAlc levels exceeded 9\%, those initiating dulaglutide had a reduction of $2.11 \%$ in $\mathrm{HbA1c}$ levels at follow-up, compared with a reduction of $1.52 \%$ in the basal insulin cohort $(\mathrm{p}<0.001)$. Overall, $22 \%$ in the dulaglutide cohort and $12 \%$ in the basal insulin cohort achieved HbA1c levels $<7 \%$ ( $<<0.002$; online appendix table 4). Similar results were obtained for patients 65 years or older at initiation: in the dulaglutide cohort, HbA1c levels dropped $1.10 \%$, compared with a decrease of $0.54 \%$ in the basal insulin group. Half of patients in the dulaglutide cohort $(50 \%)$ achieved HbA1c levels $<7 \%$, compared with $22 \%$ in the basal insulin group. Key outcomes for glycemic control using $\mathrm{mmol} / \mathrm{mol}$ units are reported in online appendix table 5 .

In the full matched population, both treatment groups exhibited significant decreases in HbAlc levels over time $(\mathrm{p}<0.01$; figure 2 , online appendix table 6). Starting at $8.65 \%$ (dulaglutide) and $8.64 \%$ (basal insulin) at baseline, HbA1c levels dropped to $7.43 \%$ in the dulaglutide cohort and $8.10 \%$ in the basal insulin cohort over the first 3 months postindex $(p<0.01)$. The reduction was durable, lasting throughout the 12-month postindex period, with HbA1c levels at 7.53\% for dulaglutide and $8.19 \%$ for basal insulin at 12 months postindex $(\mathrm{p}<0.01)$. 


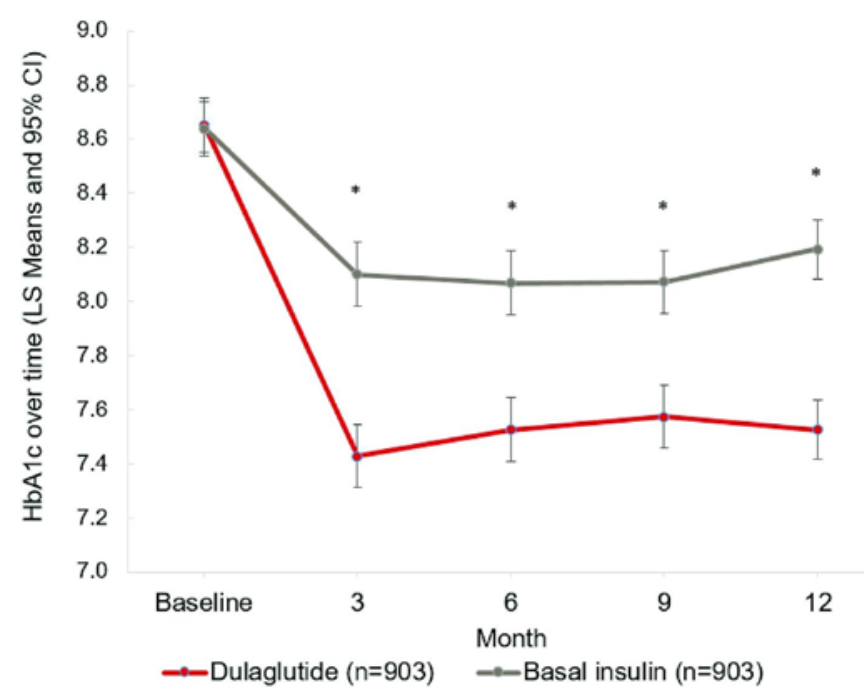

Figure 2 Longitudinal changes in $\mathrm{HbA} 1 \mathrm{c}$ among matched dulaglutide initiators versus basal insulin initiators (MMRM). *Significant difference between dulaglutide versus basal insulin with $p$ value $<0.05$. Results are from a mixed-effects model with repeated measurements (MMRM). Data represent least square mean $\pm 95 \%$ confidence limit. Baseline reflects $\mathrm{HbA1c}$ results obtained between (index date - 183 days) and (index date +14 days); the value closest to index date was chosen if there were multiple values. 3/6/9/12 months reflects $\mathrm{HbA} 1 \mathrm{c}$ results obtained between windows of \pm 45 days around index date $+92 / 183 / 274 / 365$ days, with the value closest to and prior to the anchor date chosen if there were multiple values. Point estimates are available in online supplementary appendix table 6 .

\section{Healthcare costs}

Healthcare costs were evaluated for patients with complete cost data $(n=523)$. Baseline characteristics were balanced between the two cohorts in this subgroup (online appendix table 7), and were similar to the full matched population. At 1 year postindex, patients initiating treatment with dulaglutide had significantly lower mean diabetes-related medical costs (\$3753 (SD \$9111)) than the basal insulin cohort (\$7604 (SD \$24234), $\mathrm{p}<0.001$; table 2). Mean diabetes-related pharmacy costs, however, were significantly higher for the dulaglutide cohort compared with basal insulin (\$9809 (SD \$5235) vs $\$ 6175$ (SD \$5231), $\mathrm{p}<0.001$ ). Mean diabetes-related total costs were similar between the two treatment groups ( $\$ 13$ 562 (SD \$10261) vs $\$ 13779$ (SD \$24 728); $=0.852$ ), as were mean all-cause total costs (\$20 483 (SD \$16638) vs $\$ 21718$ (SD \$38570); $\mathrm{p}=0.502$; table 2).

All-cause and diabetes-related costs per $1 \%$ HbA1c reduction were consistently lower for the dulaglutide cohort than the basal insulin cohort, with the exception of diabetes-related pharmacy costs (dulaglutide: $\$ 8174$ (SE \$504); basal insulin: \$10 292 (SE \$1768); p=0.149, figure 3 and table 2). Mean diabetes-related medical costs at 1 year follow-up per $1 \% \mathrm{HbA1c}$ reduction were \$3128 (SE \$399) for the dulaglutide cohort vs \$12673 (SE \$2825) for the basal insulin cohort. Similarly, mean total all-cause ( $\$ 17069$ dulaglutide vs $\$ 36197 ; \mathrm{p}=0.009$ ) and diabetes-related ( $\$ 11302$ dulaglutide vs $\$ 22965$ basal insulin; $p=009$ ) costs were significantly lower for the dulaglutide cohort versus basal insulin.

\section{DISCUSSION}

In this real-world study, patients initiating dulaglutide therapy had a greater reduction in glycemic levels and lower incremental cost to achieve $\geq 1 \%$ reduction in HbAlc compared with patients initiating treatment on basal insulin. Longitudinal analysis of HbA1c levels revealed differences between dulaglutide and basal insulin starting as early as 3 months that were sustained over a 1-year postindex period.

Our real-world findings are consistent with the sustained glycemic control demonstrated by dulaglutide (along with other long-acting GLP-1 RA agents) when compared with basal insulin in controlled clinical trials. An open-label randomized trial concluded once-weekly dulaglutide $1.5 \mathrm{mg}$ was associated with greater improvements in HbA1c-along with a greater proportion of patients achieving $\mathrm{HbAlc}$ levels below $7.0 \%$-and weight loss compared with insulin glargine. ${ }^{12}$ These effects persisted through the 78-week follow-up. A more recent meta-analysis of published comparisons of GLP-1 RAs to basal insulin demonstrated that although the glycemic effects differed among specific GLP-1 RAs, once-weekly agents, such as dulaglutide, produced greater reductions in $\mathrm{HbA} 1 \mathrm{c}$ levels than basal insulin. ${ }^{13}$

Published real-world research has shown that there are differences in the characteristics of patients initiating insulin compared with GLP-1 RAs: patients initiating basal insulin were more likely to be older with higher HbAlc levels, more comorbid conditions, and lower body weight compared with those initiating a GLP-1 RA. $^{6}{ }^{19}$ This underlines the importance of adjusting for patient differences when conducting comparative effectiveness research between GLP-1 RA and basal insulin. In the current analysis, prior to matching, basal insulin users were also associated with higher baseline HbAlc levels and more comorbid conditions; there were also differences in OAD use. We used PS matching to create patient cohorts with comparable baseline characteristics and in this study population, dulaglutide showed better effectiveness than basal insulin over a large range of baseline HbAlc levels. This consistent glycemic effectiveness is important considering the ADA recommendation to use GLP-1 RAs earlier in the T2D treatment algorithm. ${ }^{1}$

Previously, at least two real-world studies have compared the effectiveness of GLP-1 RAs against insulin. The first comparative study by Pawaskar et al used US electronic medical record (EMR) data from 2006 to 2009 to compare 12-month clinical outcomes of exenatide twice daily versus insulin glargine after PS matching, finding that exenatide achieved a greater mean reduction in HbAlc $(-0.6 \%$ vs $-0.4 \%) .^{7}$ The second comparative study by Overbeek $e t$ al evaluated the effectiveness of 
Table 2 Glycemic and cost outcomes at 1 year postindex among matched patients with complete cost data

\begin{tabular}{|c|c|c|c|}
\hline & $\begin{array}{l}\text { Dulaglutide initiators } \\
(n=523)\end{array}$ & $\begin{array}{l}\text { Basal insulin initiators } \\
(n=523)\end{array}$ & P value* \\
\hline \multicolumn{4}{|l|}{$\mathrm{HbA} 1 \mathrm{c}$ test results, mean (SD) } \\
\hline Baseline (\%) & $8.66(1.61)$ & $8.68(1.63)$ & 0.872 \\
\hline 1 year $(\%)$ & $7.51(1.52)$ & $8.12(1.64)$ & $<0.001$ \\
\hline 1 year change in $\mathrm{HbA} 1 \mathrm{c}$, mean (SD) & $-1.16(1.53)$ & $-0.56(1.85)$ & $<0.001$ \\
\hline \multicolumn{4}{|l|}{ Costs, mean (SD) } \\
\hline All-cause medical & $\$ 7165(\$ 13,289)$ & $\$ 12268(\$ 36,969)$ & 0.003 \\
\hline All-cause pharmacy & $\$ 13318(\$ 9,265)$ & $\$ 9450(\$ 8,945)$ & $<0.001$ \\
\hline All-cause total & $\$ 20483(\$ 16,638)$ & $\$ 21718(\$ 38,570)$ & 0.502 \\
\hline Diabetes-related medical & $\$ 3753(\$ 9,111)$ & $\$ 7604(\$ 24,234)$ & 0.001 \\
\hline Diabetes-related pharmacy & $\$ 9809(\$ 5,235)$ & $\$ 6175(\$ 5,231)$ & $<0.001$ \\
\hline Diabetes-related total & $\$ 13562(\$ 10,261)$ & $\$ 13779(\$ 24,728)$ & 0.852 \\
\hline \multicolumn{4}{|l|}{ Costs per $1 \% \mathrm{HbA} 1 \mathrm{c}$ reduction, mean (SE) } \\
\hline All-cause medical & $\$ 5971(\$ 649)$ & $\$ 20447(\$ 4,499)$ & 0.005 \\
\hline All-cause pharmacy & $\$ 11098(\$ 739)$ & $\$ 15750(\$ 2,707)$ & 0.049 \\
\hline All-cause total & $\$ 17069(\$ 1,235)$ & $\$ 36197(\$ 6,701)$ & 0.009 \\
\hline Diabetes-related medical & $\$ 3128$ (\$399) & $\$ 12673(\$ 2,825)$ & 0.004 \\
\hline Diabetes-related pharmacy & $\$ 8174(\$ 504)$ & $\$ 10292(\$ 1,768)$ & 0.149 \\
\hline Diabetes-related total & $\$ 11302(\$ 752)$ & $\$ 22965(\$ 4,125)$ & 0.009 \\
\hline
\end{tabular}

${ }^{*} \mathrm{P}$ values for $\mathrm{HbA} 1 \mathrm{c}$ and costs were obtained by t-tests. For costs, $\mathrm{p}$ values were also obtained from generalized linear model (GLM) regressions with log link and $\gamma$ distribution and with cohort as independent variable; results were similar to the t-test results. SE of the mean for cost per $1 \%$ $\mathrm{HbA} 1 \mathrm{c}$ reduction were obtained by the bootstrapping method with 5000 replications. $\mathrm{P}$ values for comparing cost per $1 \% \mathrm{HbA} 1 \mathrm{c}$ reduction among patients initiating dulaglutide versus basal insulin were obtained through t-tests using means and bootstrapped SEs.

$\mathrm{SD}$, standard deviation; SE, standard error.

liraglutide versus basal insulin in injection-naïve, obese patients with T2D from the Netherlands. ${ }^{8}$ From a baseline HbAlc of approximately $8.5 \%$, liraglutide demonstrated a statistically significant reduction in HbAlc compared with PS-matched basal insulin patients at 1year $(-1.1 \%$ vs $-0.8 \%)$. The trajectory of change in HbAlc over time with liraglutide was similar to the one seen for dulaglutide in our study, with the maximal effect as early as 3

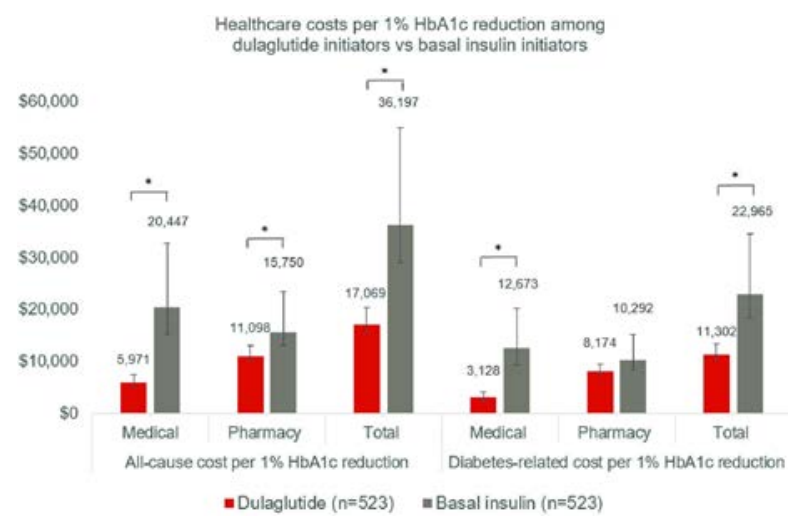

Figure 3 Healthcare costs in USD per $1 \% \mathrm{HbA} 1 \mathrm{c}$ reduction at 1 year Post-Index

*Significant difference between dulaglutide versus basal insulin with $\mathrm{p}$ value $<0.05$. Costs per $1 \% \mathrm{HbA} 1 \mathrm{c}$ reduction were calculated from the mean cost across all patients, divided by mean $\mathrm{HbA} 1 \mathrm{c}$ reduction. Error bars represent $95 \%$ Cls obtained by bootstrapping with 5000 replications. months and durability throughout 1 year. A third, noncomparative study by Wei et al examined a sample of commercially insured US patients, who were injectionnaive and initiated either liraglutide or insulin glargine. ${ }^{19}$ The study reported greater HbAlc reduction and lower cost per $1 \%$ HbAlc reduction for insulin glargine, a different finding from Overbeek ${ }^{8}$ and our current study. There were significant differences in baseline patient characteristics between the two cohorts in the Wei et al study, however, the authors stated that they were unable to adjust for these differences due to poor overlap of the PS distributions near the end points, hence subjecting comparisons across cohorts to selection bias. Additionally, the method to calculate costs per HbAlc reduction also differed from the current study. Such differences in methodology between our study and Wei et al limit comparability of results across these studies.

In patients with $\mathrm{HbAlc}>9 \%$, many physicians-especially primary care providers-often initiate basal insulin, ${ }^{9}$ while clinical guidelines recommend insulin primarily in those patients who have symptoms of hyperglycemia or catabolism, or HbA1c $>10 \% .^{2}$ In our real-world study, dulaglutide demonstrated a mean HbAlc reduction of $2.11 \%$ compared with $1.52 \%$ in the subgroup of patients with baseline $\mathrm{HbAlc}$ of $>9 \%$, providing evidence for the effectiveness of GLP-1 RAs in this subgroup of patients. In an elderly population with possible long-standing T2D, the $\beta$ cell dependent mechanism of action can also 
raise questions of effectiveness of GLP-1 RAs. However, in this study patients $\geq 65$ years old saw a similar HbAlc reduction with dulaglutide to that of the general population (and superior to basal insulin), making it a potentially effective option even for elderly patients. It is worth noting that potential weight loss associated with GLP-1 RAs should be evaluated during individualized treatment selection, especially among elderly patients.

Along with clinical effectiveness, the convenience of a treatment plays an important role, particularly in terms of adherence. ${ }^{20}$ Once-weekly administration with an easyto-use device may contribute to treatment adherence and persistence, thus resulting in better health outcomes ${ }^{9}$ which, in turn, may lead to reductions in healthcare utilization. Improved adherence is often associated with higher diabetes-related pharmacy costs. ${ }^{21}{ }^{22}$ In our study, both adherence differences and differences in acquisition costs likely affected overall diabetes-related pharmacy costs. While these costs were higher for patients receiving dulaglutide than insulin in this study, they were offset by lower medical costs and no difference was seen in overall costs. A similar pattern was observed in a previous study that examined exenatide once weekly versus insulin glargine. ${ }^{23}$ Thus, the overall economic impact of a medication must be considered in addition to medication acquisition costs.

This is also apparent in the increasing attention being paid to the affordability of medications in USA. ${ }^{24}$ To combine clinical effectiveness results with cost outcomes, our study created a basic metric that calculated the perpatient costs accrued over 1 year from treatment in relation to the change in HbAlc over that same period. We found that costs per $1 \% \mathrm{HbA1c}$ reduction were consistently lower for the dulaglutide cohort than the basal insulin cohort, with the exception of diabetes-related pharmacy costs. Presenting economic outcomes alongside clinical ones when available provides important context for decision makers. ${ }^{25}$

\section{Limitations}

Several limitations should be considered when reviewing these results. Patients were assigned to the treatment group based on their index prescription (intent-to-treat analysis) and our analysis does not account for changes in treatment during the follow-up period. However, only $10 \%-11 \%$ of patients had fills for the comparator drug over follow-up, suggesting our conclusions are not likely to be affected by these treatment changes. PS matching was used to reduce confounding of treatment selection by ensuring balance in measured baseline characteristics. However, as in other observational studies, this study was limited by the potential for bias due to unmeasured confounders, such as patient (eg, duration of diabetes, weight, education) and provider characteristics. In addition, the differences in observed patient characteristics between the unmatched cohorts imply that generalization of the clinical and economic outcomes to the full populations, especially basal insulin users with higher
HbA1c levels, is limited. Data were derived from medical and pharmacy claims, which may have contained undetected coding errors. Pharmacy claims indicate only that a prescription was filled; it is unknown whether patients used the medication as prescribed. Furthermore, pharmacy claims do not capture medications purchased in cash or over the counter. Lastly, all patients included in the study were enrolled in commercial health insurance plans in USA and satisfied all the inclusion/exclusion criteria. The results may not be generalizable to patients not selected, or with other types of health insurance or who are uninsured, or to those outside USA.

\section{CONCLUSIONS}

In this real-world study, patients with T2D who initiated dulaglutide demonstrated significantly greater and clinically meaningful HbAlc reduction compared with similar patients initiating basal insulin. This was true even in patients who initiated dulaglutide or basal insulin with a high baseline HbAlc. Although there was no significant difference in the total diabetes-related cost between the two cohorts at 1-year follow-up, the total cost per $1 \%$ HbAlc reduction was lower for dulaglutide versus basal insulin, highlighting the importance of evaluating effectiveness along with the economic impact of medications.

\section{Author affiliations}

${ }^{1}$ Global Patient Outcomes and Real World Evidence, Eli Lilly and Company, Indianapolis, Indiana, USA

${ }^{2}$ Health Economics \& Outcomes Research, HealthCore, Inc, Wilmington, Delaware, USA

${ }^{3}$ Biometrics and Advanced Analytics, Eli Lilly and Company, Toronto, Ontario, Canada

${ }^{4}$ Global Development, Eli Lilly and Company, Indianapolis, Indiana, USA

Acknowledgements The authors thank Cheryl Jones, an employee of HealthCore, for providing writing and editorial support for this manuscript.

Contributors RM, MY, HP contributed to study design, data interpretation, and manuscript drafting and review. MG and QH contributed to study design, data collection, data analysis and interpretation, and manuscript drafting and review. LW and XZ contributed to data collection, data analysis and interpretation, and manuscript review. All authors approved the final version of the manuscript before its submission.

Funding This work was supported by Eli Lilly and Company.

Competing interests RM, MY and HP are employees and stockholders of Eli Lilly and Company. MG is an employee of HealthCore, an independent research organization that received funding from Eli Lilly and Company for the conduct of this study. QH, XZ and LW were employees of HealthCore at the time the study was conducted.

Patient consent for publication Not required.

Provenance and peer review Not commissioned; externally peer reviewed.

Data availability statement № data are available.

Open access This is an open access article distributed in accordance with the Creative Commons Attribution Non Commercial (CC BY-NC 4.0) license, which permits others to distribute, remix, adapt, build upon this work non-commercially, and license their derivative works on different terms, provided the original work is properly cited, appropriate credit is given, any changes made indicated, and the use is non-commercial. See: http://creativecommons.org/licenses/by-nc/4.0/.

ORCID iD

Michael Grabner http://orcid.org/0000-0002-6521-1007 


\section{REFERENCES}

1 American Diabetes Association. 9. Pharmacologic Approaches to Glycemic Treatment: Standards of Medical Care in Diabetes-2019. Diabetes Care 2019;42:S90-102.

2 Garber AJ, Abrahamson MJ, Barzilay JI, et al. Consensus statement by the american association of clinical endocrinologists and american college of endocrinology on the comprehensive type 2 diabetes management algorithm - 2019 EXECUTIVE SUMMARY. Endocr Pract 2019;25:69-100.

3 Abd El Aziz MS, Kahle M, Meier JJ, et al. A meta-analysis comparing clinical effects of short- or long-acting GLP-1 receptor agonists versus insulin treatment from head-to-head studies in type 2 diabetic patients. Diabetes Obes Metab 2017;19:216-27.

4 Brod M, Kongsø JH, Lessard S, et al. Psychological insulin resistance: patient beliefs and implications for diabetes management. Qual Life Res 2009;18:23-32.

5 Trujillo JM, Nuffer W, Ellis SL. Glp-1 receptor agonists: a review of headto-head clinical studies. Ther Adv Endocrinol Metab 2015;6:19-28.

6 Yu M, Mody R, Landó LF, et al. Characteristics associated with the choice of first injectable therapy among US patients with type 2 diabetes. Clin Ther 2017;39:2399-408.

7 Pawaskar M, Li Q, Hoogwerf BJ, et al. Metabolic outcomes of matched patient populations initiating exenatide Bid vs. insulin Glargine in an ambulatory care setting. Diabetes Obes Metab 2012;14:626-33.

8 Overbeek JA, Heintjes EM, Huisman EL, et al. Clinical effectiveness of liraglutide vs basal insulin in a real-world setting: evidence of improved glycaemic and weight control in obese people with type 2 diabetes. Diabetes Obes Metab 2018;20:2093-102.

9 Anderson JE, Thieu VT, Boye KS, et al. Dulaglutide in the treatment of adult type 2 diabetes: a perspective for primary care providers. Postgrad Med 2016;128:810-21.

10 Matfin G, Van Brunt K, Zimmermann AG, et al. Safe and effective use of the once Weekly Dulaglutide single-dose Pen in InjectionNaïve patients with type 2 diabetes. J Diabetes Sci Technol 2015;9:1071-9.

11 Yu M, Van Brunt K, Milicevic Z, et al. Patient-Reported outcomes with once Weekly dulaglutide versus placebo, both in combination with once daily insulin glargine (+/- metformin) in type 2 diabetes (AWARD-9). Munich, Germany: 52nd European Association for the Study of Diabetes (EASD) Annual Meeting, 2016.

12 Giorgino F, Benroubi M, Sun J-H, et al. Efficacy and safety of onceweekly dulaglutide versus insulin Glargine in patients with type 2 diabetes on metformin and glimepiride (AWARD-2). Diabetes Care 2015;38:2241-9.

13 Singh S, Wright EE, Kwan AYM, et al. Glucagon-Like peptide-1 receptor agonists compared with basal insulins for the treatment of type 2 diabetes mellitus: a systematic review and meta-analysis. Diabetes Obes Metab 2017;19:228-38.

14 Quan $\mathrm{H}$, Sundararajan V, Halfon $\mathrm{P}$, et al. Coding algorithms for defining comorbidities in ICD-9-CM and ICD-10 administrative data. Med Care 2005;43:1130-9.

15 Chang H-Y, Weiner JP, Richards TM, et al. Validating the adapted diabetes complications severity index in claims data. Am J Manag Care 2012;19:721-6.

16 Austin PC. Optimal caliper widths for propensity-score matching when estimating differences in means and differences in proportions in observational studies. Pharm Stat 2011:10:150-61.

17 Polsky D, Glick HA, Willke R, et al. Confidence intervals for costeffectiveness ratios: a comparison of four methods. Health Econ 1997;6:243-52.

18 DeSouza CM, Legedza ATR, Sankoh AJ. An overview of practical approaches for handling missing data in clinical trials. J Biopharm Stat 2009;19:1055-73.

19 Wei W, Buysman E, Grabner M, et al. A real-world study of treatment patterns and outcomes in US managed-care patients with type 2 diabetes initiating injectable therapies. Diabetes Obes Metab 2017;19:375-86.

20 Carls GS, Tuttle E, Tan R-D, et al. Understanding the gap between efficacy in randomized controlled trials and effectiveness in realworld use of GLP-1 RA and DPP-4 therapies in patients with type 2 diabetes. Diabetes Care 2017;40:1469-78.

21 Kennedy-Martin T, Boye K, Peng X. Cost of medication adherence and persistence in type 2 diabetes mellitus: a literature review. Patient Prefer Adherence 2017;11:1103-17.

22 Lee WC, Balu S, Cobden D, et al. Prevalence and economic consequences of medication adherence in diabetes: a systematic literature review. Manage Care Interface 2006;19:31-41.

23 Wittbrodt E, Kong AM, Moore-Schiltz L, et al. All-Cause and diabetes-related healthcare costs among US adults with type 2 diabetes initiating exenatide once Weekly or insulin glargine. Diabetes Obes Metab 2018;20:672-80.

24 Schaffer SK, Messner D, Mestre-Ferrandiz J, et al. Paying for Cures: Perspectives on Solutions to the "Affordability Issue". Value Health 2018;21:276-9.

25 The AMCP format for formulary submissions: welcome to version 4.0. J Manag Spec Care Pharm 2016;22:444-6. 